\title{
Eating disorder services for young people in Ireland: Perspectives of service providers, service users and the general adolescent population
}

\author{
Fiona McNicholas ${ }^{1}$, Cliodhna O'Connor ${ }^{2}$, Niamh McNamara ${ }^{3}$, Lesley O'Hara ${ }^{1}$ \\ ${ }^{1}$ School of Medicine, University College Dublin, Ireland \\ ${ }^{2}$ Department of Psychology, Maynooth University, Ireland \\ ${ }^{3}$ Division of Psychology, Nottingham Trent University, UK
}

Article published in the Irish Journal of Psychological Medicine

Citation: McNicholas, F., O’Connor, C., McNamara, N., \& O'Hara, L. (2015). Stigma and treatment of eating disorders in Ireland: Health professionals' knowledge and attitudes.

Irish Journal of Psychological Medicine. Advance online publication. doi:

10.1017/ipm.2015.66

Final version available at:

http://journals.cambridge.org/action/displayAbstract?fromPage=online\&aid=10079097\&fullt extType=RA\&fileId=S07909667150006 


\begin{abstract}
Objectives: This paper illuminates how national eating disorder (ED) policy translates into day-to-day practice by exploring how ED services are experienced by those who deliver and use them.
\end{abstract}

Methods: A mixed-methods approach was used, which combined qualitative and quantitative techniques. The paper collates data from three studies: (i) an interview study exploring the lived experiences of young people with EDs $(n=8)$, their parents $(n=5)$ and their healthcare professionals ( $n=3$ ); (ii) a national survey of health professionals' perspectives on existing ED services ( $n=171)$; (iii) a nationwide survey of secondary-school students' eating concerns and patterns of help-seeking $(n=290)$.

Results: The qualitative interviews with young people and their parents revealed feelings of isolation and helplessness. Young people expressed interest in patient support groups, while parents desired greater support for the family unit. Parents were highly critical of available services, particularly in relation to access. These criticisms were echoed in the survey of healthcare professionals, who reported many barriers to delivering effective care. Clinicians were almost unanimous in calling for care pathways to be clarified via a standardised treatment protocol. The survey of adolescents indicated widespread reluctance to seek help regarding eating concerns: over one-third expressed concern about their own eating habits, but half of these had not divulged their concerns to anyone. Participants' preferred pathways of help-seeking revolved around family and friends, and adolescents were unsure about routes of access to professional support. 
Conclusions: The research demonstrates that many aspects of national ED policy have not been implemented in practice. The paper highlights specific gaps and suggests ways they can be redressed.

\section{Keywords}

Eating disorders; mental health services; youth mental health; surveys; interviews 


\section{Introduction}

It is estimated that up to 200,000 people in Ireland are affected by eating disorders (EDs), with 400 new cases emerging each year (Department of Health \& Children, 2006). EDs represent a particular significant portion of the mental illness burden of young people, accounting for $12 \%$ of all juvenile admissions to Irish psychiatric units (Health Research Board, 2014). However, the most recent expert review of ED service provision in Ireland characterised services as "very poorly developed" (Department of Health \& Children, 2006, p. 76). The current paper enhances understanding of the day-to-day operation of ED services by exploring how they are experienced by those who deliver and use them. By compiling quantitative and qualitative data from health professionals and existing and potential patients, the paper furnishes a valuable insight into how ED policy is implemented in practice.

\section{ED policy in Ireland}

The 2006 expert review of national mental health policy, A Vision for Change (AVFC), identified numerous weaknesses in ED service provision. These included an inadequate national stock of in-patient beds; little cohesion between child and adolescent (CAMHS) and adult mental health services (AMHS); insufficient attention to EDs in medical education and resultant deficiencies at primary care level; and a need for greater public awareness of EDs in the wider community. $A V F C$ recommended a number of measures to address these problems, including coordinating services regionally, public education campaigns and improved training of staff.

$A V F C$ was launched with a planned implementation period of 7-10 years (i.e. recommendations actualised by 2013-2016). Unfortunately, the College of Psychiatry of Ireland's subsequent progress reports suggest that service provision appears to be moving further away from, rather than closer to, compliance with the report's recommendations (College of Psychiatry of Ireland, 2010). For example, no progress has been achieved towards 
establishing a national tertiary referral centre or attaining the recommended quantity of publicly-available ED beds (College of Psychiatry of Ireland, 2012).

The importance of the development of Ireland's ED policy was further underlined by its inclusion as one of the first three National Clinical Programmes for Mental Health (Health Service Executive, 2011). Like $A V F C$, this programme stresses early identification whereby primary care practitioners, supported by voluntary organisations, can detect disordered eating and intervene in mild-to-moderate cases. It expands on the recommendations of $A V F C$ by recommending specific community-level education and prevention initiatives, primary carelevel screening of at-risk populations, and reduced waiting times. However, despite some dedicated training, progress has been slow.

\section{From policy to practice: The STEDI project}

Inspection of official ED policy is of limited use in understanding the realities of service delivery, since research shows there is often a wide gap between the evidence-based procedures enshrined in policy documents and actual clinical practice (Procter et al., 2009). Data illuminating how national ED policy translates into the day-to-day delivery of care is very sparse. A 2006 survey of 91 Irish general practitioners found that GPs had lower caseloads of EDs than epidemiological data would predict, did not routinely screen for EDs, and had low levels of confidence in their ability to manage ED treatment (Flahavan, 2006). Participants criticised the poor referral options available to them and difficulties with accessing ED services. Beyond Flahavan (2006), no research has explored whether GPs' attitudes have changed since the publication of $A V F C$, or investigated ED services from the perspective of other health professionals, patients or their families.

The current paper seeks to remedy this gap by providing a holistic insight into how ED services are perceived and experienced by medical practitioners, service-users and the general 
public. To achieve this, it utilises a mixed-methods approach that combines qualitative and quantitative data analysis techniques. This approach is often recommended in health services research because it promotes a more comprehensive, multidimensional and contextualised analysis (O'Cathain, Murphy \& Nicholl, 2007). It combines rich insight into people's lived experience with the reliability of rigorous quantitative methods that access a larger section of the population.

This paper mobilises data from three studies undertaken as part of the STEDI (Stigma and Treatment of Eating Disorders in Ireland) research programme.

i. a multi-perspective interview study exploring the lived experiences of young people with EDs, their parents and their healthcare professionals;

ii. a national survey of health professionals' experience of delivering ED care and perspectives on existing ED services;

iii. a nationwide survey of the general population of secondary school students, exploring their experiences of eating and body image concerns and patterns of helpseeking.

This paper extracts the data from each of these studies that pertain to participants' views and experience of ED services. It will describe the methodology and results of each study in turn, before closing with a general discussion of their cumulative implications.

\section{Study 1: Qualitative study of service-users' experiences}

The first study adopted a qualitative approach to enlighten the perspective of service-users and their families. 


\section{Study 1 methodology}

\section{Participants}

Potential participants were alerted to the study by clinicians who had been sent information leaflets or by media advertisements. Eight adolescents (six female, two male; aged 15-19, modal age 15) opted into the study. At the time of interviews, participants had held a diagnosis of Anorexia Nervosa (AN) or Bulimia Nervosa (BN) for an average of 9.9 months (range 3-14 months).

\section{Design}

In-depth, semi-structured interviews were conducted with the adolescents, their parents (five mothers alone; one father alone; and both parents of one participant) and three professionals involved in their care (the GP and psychologist of a 16 year-old boy with AN, and the GP of 16 year-old girl with AN). Interview questions were broad and open-ended, to afford participants the opportunity to articulate their individual experiences without the restrictions of a formalised interview schedule. Interviews took place between October-July 2013 in locations convenient for participants (usually their homes). After the interviews, audio files were transcribed and anonymised. The data were analysed using a grounded theory approach (Strauss \& Corbin, 1990) to illuminate how ED services were experienced through the eyes of participants.

\section{Study 1 results}

\section{Previous service engagement}

Of the young persons (YPs) who participated, five received CAMHS intervention only. One participant received private (inpatient) treatment only and demonstrated unawareness that public treatment options were available. The remaining two participants had attended both CAMHS and private care. Of these, one boy with AN sought private care until it became 
financially unsustainable, at which point his GP referred him to CAMHS. The family of the other YP with experience of both services was dissatisfied with the waiting times and physical appearance of CAMHS and opted to continue private treatment, despite financial burden and a six-hour roundtrip.

\section{Young people's perspectives on treatment and services}

Generally, YPs who received CAMHS care were satisfied with the services they received. However, some resented feeling treated as passive recipients of care. In particular, they objected to instances where clinicians presumed that they wanted to overcome their ED, and ignored the YP's own ambivalence about recovery. YPs felt this marginalisation of their own perspective undermined the potential efficacy of treatment.

they just take it as like I want to get better, they don't really ask me. So when they say like oh you've lost weight this week or you've gained weight this week, they don't really say like... do you actually want this. [...] So sometimes I'm like, maybe I'm just wasting it all like, going here and maybe I should just get proper help when I think I will use the help. [YP1]

Unanimously, participants expressed a desire for contact with others affected by EDs and the provision of support groups which would allow them to feel 'understood'.

Support groups. [...] it's someone who I can talk to who is going through what I'm going through, they understand. Like the doctors that I talk to, they know, they've studied it but they don't really know what I'm going through, they only have an idea. [YP3] 
Specifically, participants anticipated that interactions with people more advanced in the recovery process would increase their commitment to recovery, by transforming it from a lofty concept to a realistically attainable objective.

\section{Parents' perspectives on treatment and services}

Parents did not share the YPs' general satisfaction with services. Overwhelmingly, parents described feeling alone and unsupported in their carer role. They described feelings of hopelessness and frustration as they struggled to give their child the care they required, while simultaneously caring for their rest of their family.

I mean it affects everybody... it was very stressful, like it was really stressful... and it's just like, just feels so like nobody will help her [YP1:Mother]

Parents felt forced into a position for which they were unqualified, and desired reassurance from health professionals that they were 'doing it right'. For many parents, their own wellbeing suffered due to the demands of their child's condition. Their attempts to secure support for this were typically unsuccessful.

there is no care, there's no outlet or outreach that we could go, like I tried myself to try and get somewhere, there is nowhere... there is no help. [YP3:Mother]

Parents expressed particular frustration at the delay in obtaining a CAMHS appointment following referral. Some parents explained that their child's condition deteriorated so much during this time that their GP referred them for emergency intervention in hospitals or recommended they seek private care if they could afford it.

\section{Health professionals' perspectives on treatment and services}

While only three health professionals took part in the study, their experiences offer a useful insight into how services are experienced by those tasked with delivering them. Both GPs 
who participated felt that they did not have the necessary expertise to treat EDs themselves, and complained of a lack of referral options. Echoing parents, GPs expressed frustration at the length of time waiting for CAMHS appointment. For the participant within the CAMHS service (a psychologist), staffing and resourses were identified as the greatest obstacle to caring for YPs with EDs.

\section{Summary}

The interviews with YPs, parents, and health care professionals point to the need for greater peer support, psycho-education and service resources. YPs, whose ED induced a sense of isolation, believed that their well-being and recovery would be facilitated by the provision of support groups where they could engage with other people affected by ED. Like their children, parents felt alone and unsupported, and unqualified to meet the needs of their child. They called for greater acknowledgement that EDs are an issue that affects the whole family unit, and for services to include whole-family support. The interviews with healthcare providers reiterated the flaws in existing service provision, with limitations of expertise and resources hampering clinicians' ability to provide effective care.

\section{Study 2: Survey of health professionals}

The second element of the project surveyed health professionals' perspective on ED services.

\section{Study 2 methodology}

\section{Participants}

The contact details of a purposive sample of 1,916 health professionals were obtained through national representative bodies (Irish Medical Directory; consultant lists for CAMHS and AMHS; Heads of Psychology Services Ireland; Irish Association for Counselling and Psychotherapy; and the Irish Nutrition \& Dietetic Institute). Each clinician was issued survey invitations and follow-up reminders by email. In total, 171 responded (response rate 9\%). The 
sample was composed of psychiatrists $(N=30,20 \%)$, psychologists $(N=21,14 \%)$, counsellors $(N=60,40 \%)$ and other professions $(N=16,11 \%)$ including nurses, dieticians and social workers. Participants ( $72 \%$ female) had an average of 15.8 years of clinical experience $(S D=$ 9.2).

\section{Design}

Consenting participants completed the survey online using the SurveyMonkey software between October 2013 and February 2014. In addition to questions assessing health professionals' attitudes toward and knowledge about EDs (described in McNicholas, O’Connor, O’Hara \& McNamara, 2015), it also covered typical treatment practice, estimated numbers of EDs seen annually, participants' personal experiences with disordered eating, and participants' evaluations of existing services and voluntary organisations. Quantitative data was subjected to statistical analysis using SPSS and free-text responses were analysed using content analysis techniques. In the statistical analyses below, missing cases were excluded pairwise and percentage figures are derived from the subset of the sample who responded to that item.

\section{Study 2 Results}

\section{Professional experience of EDs}

Of the 90 participants who provided information about their professional experience with EDs, the vast majority $(94 \%, N=85)$ reported previously treating an individual ( $86 \%$ female) with an ED. Participants had an average of $2.36(S D=4.1)$ cases in current treatment. Table 1 shows that $\mathrm{AN}$ was the ED subtype most frequently encountered in clinical practice and that individuals within the professional category of 'other' encountered most cases of ED, followed by psychiatrists and GPs. 
Table 1. Mean number of 15-18 year old patients with EDs seen in previous 12 months

\begin{tabular}{|c|c|c|c|c|c|c|c|c|c|c|}
\hline & \multicolumn{2}{|c|}{$\begin{array}{l}\text { Anorexia } \\
\text { Nervosa }\end{array}$} & \multicolumn{2}{|c|}{$\begin{array}{l}\text { Bulimia } \\
\text { Nervosa }\end{array}$} & \multicolumn{2}{|c|}{$\begin{array}{c}\text { Binge Eating } \\
\text { Disorder }\end{array}$} & \multicolumn{2}{|c|}{$\begin{array}{l}\text { Eating Disorder } \\
\text { Not Otherwise } \\
\text { Specified }\end{array}$} & \multicolumn{2}{|c|}{ Total } \\
\hline & $M$ & $S D$ & $M$ & $S D$ & $M$ & $S D$ & $M$ & $S D$ & $M$ & $S D$ \\
\hline 'Other' & 3.5 & 3.45 & 0.6 & 0.84 & 0.56 & 1.13 & 2.9 & 4.84 & 7.11 & 10.35 \\
\hline Psychiatrists & 2.74 & 3.12 & 0.87 & 1.01 & 0.29 & 0.56 & 1.32 & 2.32 & .25 & 6.17 \\
\hline GPs & 1.41 & 1.32 & 0.79 & 0.8 & 0.54 & 0.88 & 0.88 & 1.08 & 3.46 & 3.1 \\
\hline Psycholog & 1.11 & 1.54 & 0.14 & 0.38 & 0 & 0 & 0.5 & 0.54 & 1.57 & 2.15 \\
\hline Counsellors & 0.6 & 0.91 & 0.72 & 1.6 & 0.62 & 0.8 & 0.32 & 0.57 & 1.55 & 1.87 \\
\hline Total & 1.77 & 2.44 & 0.71 & 1.14 & 0.45 & 0.77 & 1.06 & 2.28 & 3.65 & 5.42 \\
\hline
\end{tabular}

When asked whether they had noticed any recent changes in presentations of EDs, 60 (67.4\%) had not observed any while 29 (32.6\%) had. An open question asked participants to describe, in their own words, the changes they had noticed. Figure 1 shows that the most common perceived changes were a younger age of onset and an increase in male presentations.

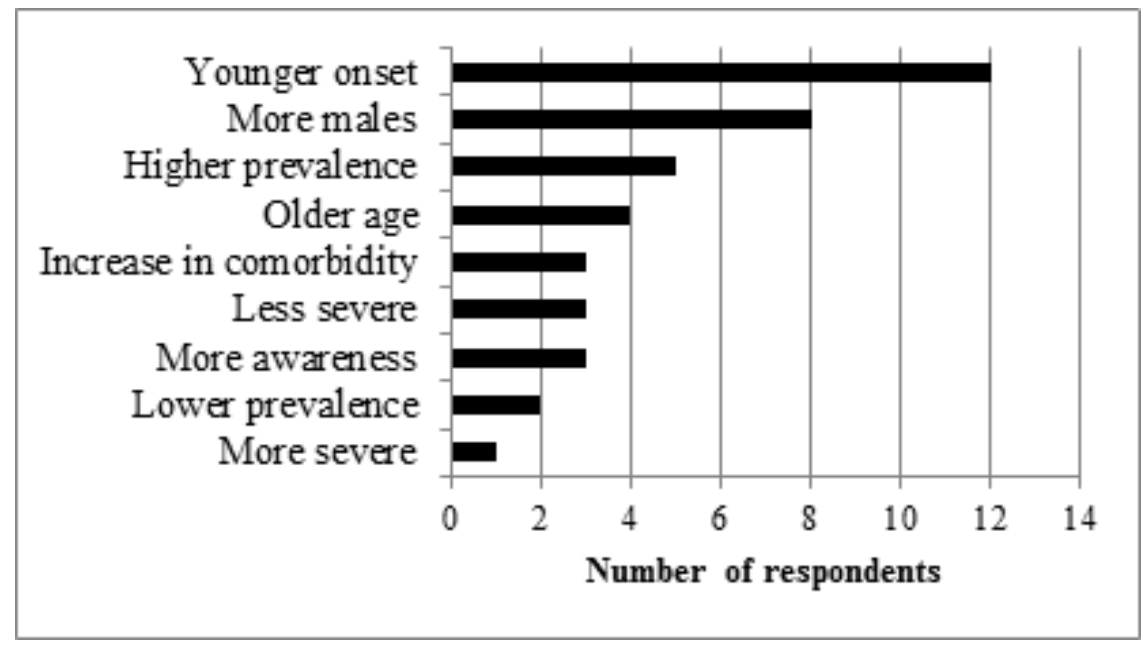

Figure 1. Most common responses in open question regarding recent changes observed in ED cases

\section{Appraisal of services}

Seventy-nine participants responded to another open question asking participants to describe their experience of ED services, with most comments being critical in nature (Figure 2). Echoing the findings of Study 1, particular foci of criticism were the limited geographic 
distribution of services, inadequate provision of specialist services and difficulties accessing services (particularly for older adolescents).

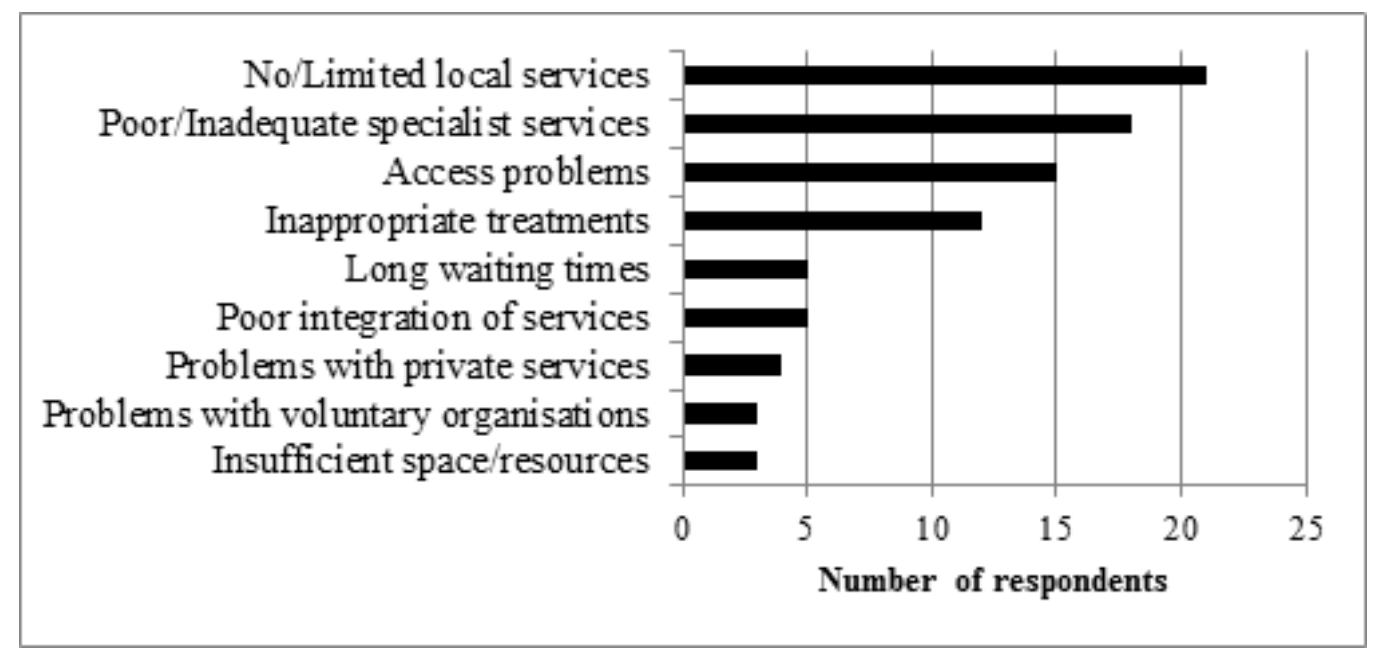

Figure 2. Negative comments about existing services

Sixteen participants offered positive comments about existing services, applauding the quality of services experienced $(N=11)$, especially private services $(N=5)$, and ease of access $(N=5)$.

The vast majority of respondents $(N=81,90 \%)$ indicated that they would benefit from the publication of a standardised protocol for treatment of EDs.

\section{Voluntary organisations}

The majority $(80 \%)$ of respondents stated that they were aware of voluntary organisations, with Bodywhys most often named $(N=57)$. Voluntary organisations were primarily perceived as a source of support/advice and information, with little mention of their role in advocacy or research. When asked if they had ever referred a patient to a voluntary organisation, only $63 \%(N=55)$ of those who answered indicated that they had.

\section{Summary}

This sample of health professionals indicated that they encountered an average of four patients with EDs annually, predominantly females with AN. Some reported a recent increase 
in younger and male patients. Clinicians expressed a need for clearer guidance for dealing with ED patients, and were almost unanimous in calling for a standardised treatment protocol. The vast majority were highly critical of the quality of existing ED services, with fewer than one in ten offering any positive comment on services. While most clinicians were aware of voluntary support organisations, considerably fewer had referred patients to these services.

\section{Study 3: Survey of adolescents}

The final component of this project examined adolescents' understanding of existing support structures and delivery preferences.

\section{Study 3 Methodology}

\section{Participants}

Two hundred and sixty Irish secondary schools were randomly selected from Department of Education \& Skills lists and invited to participate in the research. Sixty-three schools (24\% response rate) agreed to recruit students to the study. Schools arranged the collection of signed consent from parents and assent from students. Responses were obtained from 290 participants (51.1\% male, mean age 16.8, range $15-19)$. Just under half $(46.3 \% ; N=132)$ were enrolled in all-girls schools, 20.7\% (N=59) attended all-boys schools and 33\% (N=94) attended mixed-gender schools. Approximately one-quarter $(26.1 \% ; N=74)$ attended feepaying schools. This is a reasonably good representation of the national post-primary school system (Department of Education \& Skills, 2015).

\section{Design}

A survey was constructed and made available in both paper and electronic form (on the SurveyMonkey website) between March-October 2014. Demographic and educational characteristics preceded questions assessing participants' attitudes and experience regarding 
EDs, levels of body satisfaction, knowledge of how to access ED information and health services, and preferred help-seeking behaviours. Data was subjected to statistical analysis using SPSS.

\section{Study 3 Results}

\section{Body image and disordered eating}

Of the 186 who answered a question about whether they knew someone affected by an ED, the majority $(62.4 \% ; N=116)$ responded that they did: 6 indicated themselves, 14 a member of their immediate family, 53 a close friend, 9 a member of their extended family, and 36 an acquaintance.

Participants rated their body satisfaction on a 5-point scale $(M=2.94, S D=1.51)$. Only $4.6 \%$ of participants rated themselves as 'very satisfied' and $38.5 \%$ as 'satisfied' with their body. There was a significant effect of gender, $t(256)=-6.16, p<.001$, with boys rating themselves more satisfied $(M=3.36, S D=1.05)$ than girls $(M=2.53, S D=1.1)$.

\section{Eating concerns and their disclosure}

When asked if they were concerned about their own eating habits, 9.7\% (N=28) declined to answer this question. Of those who responded, most $(N=163 ; 62.2 \%)$ denied any concern. Approximately one-third $(N=99 ; 60$ female, 27 male, 2 unspecified $)$ reported being concerned, of whom half $(N=51)$ had discussed these concerns with someone else. This was usually a family member $(N=30)$ or friend $(N=17)$; only three had consulted a health professional and one a support organisation. Of the 59 who elaborated on their reasons for lack of disclosure, 32.2\% (N=19) expressed a fear of being judged, 27.1\% $(N=16)$ that their concerns were not sufficiently serious, and $16.9 \%(N=10)$ that they would not know who to tell. 


\section{Help-seeking preferences}

Participants were asked to rate on a 4-point scale their likelihood of seeking help from a range of sources, in the event that they developed eating concerns. A principal components analysis with Varimax rotation was performed on this data, and a scree plot indicated that a three-factor solution was appropriate. The three-factor solution accounted for $60.7 \%$ of the total variance. Four items referring to expert help (health professional, email/online counselling, telephone counselling, Bodywhys) loaded onto the first factor $(21.27 \%$ variance), four items relating to personal contacts (parent, friend, family friend, relative) loaded onto the second factor $(20.19 \%$ variance $)$, and three items relating to impersonal information-providers (book/magazine, internet website, social network site) loaded onto the final factor (19.22\% variance). The items corresponding to each factor were summed to create three composite variables labelled 'Expert' $(\alpha=.73)$, 'Personal' $(\alpha=.68)$ and 'Media' $(\alpha=.69)$. Respondents rated Personal help highest $(M=2.51, S D=.63)$, followed by Media $(M=2.23, S D=.73)$ and Expert $(M=2.15, S D=.65)$ help.

A two-way mixed ANOVA was performed to assess the influence of type of help and personal eating concern on the likelihood of seeking help. The analysis showed a significant main effect of personal eating concern: on average, participants who had reported being concerned about their own eating habits were significantly less likely to seek help, $F(1,243)=5.77, p=.017$. The results also showed a significant main effect of the type of help, $F(2,486)=21.65, p<.001$. Post-hoc pairwise comparisons with Bonferroni corrections showed that on average, personal advice was significantly preferred to both media advice $(p<.001)$ and expert advice $(p<.001)$ with no significant difference between the latter two $(p=.081)$. Finally, there was a significant interaction between type of help and personal eating concern, $F(2,486)=5.16, p=.006$, whereby those who reported eating concerns were less likely to seek 
personal and expert advice than the unconcerned participants, but about equally likely to consult media sources.

\section{Understanding of available supports}

Four items assessed participants' self-rated understanding of how to seek help for eating problems, on a five-point scale from 'not good at all' to 'excellent'. Table 2 shows that all mean scores were below the scale mid-point, indicating poor understanding of help-seeking options. Participants' confidence that they would know how to obtain access to a health professional was particularly low.

Table 2. Mean scores for understanding of help-seeking options

\begin{tabular}{lll}
\hline & $M$ & $S D$ \\
\hline Who to talk to if you were worried about your eating habits & 2.97 & 1.17 \\
Where else to look for information on eating problems & 2.93 & 1.19 \\
How to help a friend who is worried about their eating habits & 2.85 & 1.25 \\
How to access a health professional & 2.68 & 1.29 \\
\hline
\end{tabular}

\section{Summary}

Many adolescents had personal acquaintance with individuals with EDs and over one-third indicated a personal concern about their own eating habits, but half of these had not divulged their concerns to anyone. Participants with personal eating concerns were least likely to seek help. Participants' preferred pathways of help-seeking for eating concerns revolved mainly around personal contacts, such as family and friends. However, peers may not be an effective route of help-seeking, given that this sample reported poor understanding of the supports available for eating problems. Their particularly low confidence that they would know how to obtain professional help for eating concerns indicates a specific gap in awareness about routes of access to mental health services.

\section{Discussion}

The STEDI project provides a valuable glimpse into the actual implementation and delivery of ED services in Ireland, from the perspective of young people, their parents, and health 
professionals. It provides empirical evidence that many of the recommendations in national ED policy have not been implemented, with potentially serious implications for those using and delivering ED services.

Consistent with other studies (McNicholas et al., 2010), personal dissatisfaction with eating and body image was high in the adolescent survey, suggesting that disordered eating continues to be a serious risk for this cohort. This problem is further exacerbated by two particular issues identified by this research. Firstly, detection of emerging EDs is likely to be undermined by the fact that many young people worried about their eating conceal their concerns, often due to apprehension of negative judgement. This highlights the importance of encouraging early disclosure of eating-related concerns and of fostering a non-judgemental attitude to EDs in clinical settings and society at large. Secondly, young people do not feel they have a good understanding of how to access support for eating problems. Participants' preferred pathways of help-seeking revolve mainly around personal contacts such as family and friends, and these authentic social networks represent valuable resources for mental health campaigns to harness. However, such measures will not be effective unless the general public is equipped with the knowledge and tools to support young people who express eating concerns: soliciting advice from someone with a poor understanding of EDs may hinder rather than help the recovery process. Young people's particularly low confidence that they would know how to obtain professional help for eating problems indicates a specific need for greater dissemination of information about routes of access to mental health services.

Ambiguity about access to ED services was an issue that arose across all three studies. Consistent with Flahavan (2006), the survey of health professionals elicited many critical comments about access-related issues, including the limited geographic distribution of specialist services, long waiting times after initial referral, inadequate provision for 16-18 year olds, and the severely restricted resources of public ED services. All of these issues were 
corroborated by the health professionals and parents interviewed in the qualitative study. Both parents and clinicians criticised the delays in accessing CAMHS services and the necessity of reliance on financially unsustainable private treatment options. General confusion about access also became anecdotally apparent while completing the research: after publicising the call for participants, the research team were contacted by several parents, GPs and people with undiagnosed EDs, all seeking information about how to access services. The expansion and streamlining of access to ED services is clearly an issue in need of attention.

Beyond questions of access, a further issue highlighted by the qualitative study in particular was patients' and parents' unmet needs for emotional and social support. Young people with EDs unanimously called for support groups that would address their sense of isolation and provide models for successful recovery. Parents also experienced a lack of outlets in which they could express and find support for their feelings of loneliness and hopelessness. Parents indicated that although EDs impacted on the entire family, this was often not addressed in treatment. The dearth of family therapy and support services available in Ireland runs counter to international best practice in ED treatment, which recommends that family members (including siblings) be included in treatment programmes that involve "the sharing of information, advice on behavioural management, and facilitating communication" (NICE, 2004, p.4). Such interventions both equip families with the tools to support the young person's recovery, and combat parents' own sense of helplessness and isolation. This study provides evidence supporting the roll-out of Family-Based Treatment for ED that is currently underway within the HSE.

The survey of health professionals showed that clinicians recognised the need for greater support of patients and families, but felt unable to provide this due to resource constraints. Voluntary organisations are ideally placed to supplement strained clinical resources, as acknowledged in both $A V F C$ and the National Clinical Programme for Mental Health. 
However, although most health professionals who responded to the survey were aware of voluntary organisations that specialise in EDs, only a minority of clinicians reported referring to these groups. There is clearly room for increased collaboration between clinical and voluntary services. However, the development of a defined role for voluntary services in ED prevention and treatment programmes is contingent on the tangible support promised to voluntary services in $A V F C$.

Finally, the research provides much-needed data on the prevalence of EDs in Irish health professionals' caseloads. In the year prior to the survey, participants reported that they had treated an average of four young people affected by an ED. Consistent with previous data from Ireland (Flahavan, 2006) and the UK (Royal College of Psychiatrists, 2012), AN was the ED most frequently encountered in clinical practice. However, epidemiological data shows that $\mathrm{BN}$ and Binge Eating Disorder (BED) are considerably more prevalent than $\mathrm{AN}$ in the general population. The discrepancy in prevalence rates in community and clinical settings is consistent with evidence that health services are disproportionately oriented towards AN (Royal College of Psychiatrists, 2012) and indicates a particular problem with underdetection of $\mathrm{BN}$ and BED. Efforts should be made to heighten awareness of these disorders among clinical professionals and the general population.

It is important to acknowledge the limitations of the current research. Firstly, despite extensive efforts to recruit participants, the sample sizes for each of the three studies were lower than desired. Future research should be conducted to determine whether the findings generalise to larger samples. In particular, the $9 \%$ response rate in Study 2 introduces the possibility of selection bias: perhaps those who responded had a distinctive interest in the topic. Nevertheless, low response rates are typical in surveys of time-pressed health professionals (Aitken, Power, \& Dwyer, 2008), and the sample size is considerably higher than the only previous Irish study on this topic (Flahavan, 2006). Secondly, the survey of the 
general population only targeted young people. Although this is the age-period at which most EDs develop, many EDs are lifelong conditions, as indeed was acknowledged by the adolescents interviewed in the qualitative study. Additionally, the low ED caseloads reported by the health professionals suggest that many EDs can go undetected in adolescence, therefore persisting into adulthood. Further research should be conducted to assess the services available for the detection and treatment of EDs across the life course. Finally, this research took place in 2013-2014, a time when Irish health services were still feeling the repercussions of the financial crisis and subsequent austerity measures, and in advance of the National Clinical Programme for EDs. A longitudinal study of how ED services have expanded or restricted over time, and the implications for levels of risk and morbidity, would furnish a valuable evidence-base for future policy development.

The data collected by the STEDI project demonstrates that in the nine years since the publication of $A V F C$, service provision for EDs has remained substandard. The research shows that these deficiencies have resulted in a situation where service-users and their families feel unsupported and misunderstood; where health professionals feel ill-equipped to perform their assigned roles; and where young people struggle in silence with concerns relating to body image and eating, not knowing how to access help. A structured programme for the implementation and auditing of effective ED services is urgently required to mitigate the serious psychological, social and economic consequences that EDs impose on young people and the country as a whole.

\section{Financial Support}

This research was made possible through a grant from the Saint John of God Research Department (grant ref. 512). 


\section{Conflict of Interest}

The authors have no conflict of interest to report.

\section{Ethical Standards}

The research received ethical approval from the Saint John of God Research Ethics Committee and complied with all relevant ethical standards. Participants gave informed consent to participate and were fully debriefed as to the purpose of the research.

\section{References}

Aitken C, Power R, Dwyer R (2008). A very low response rate in an on-line survey of medical practitioners. Australian and New Zealand Journal of Public Health 32, 288-289.

College of Psychiatry of Ireland (2010). The College of Psychiatry of Ireland submission to the 2nd A Vision for Change Monitoring Group.

(http://www.irishpsychiatry.ie/Libraries/External_Events_Documents/College_report_to_the _2nd_Indep_monitoring_group_on_AVFC_progress_Nov_2010.sflb.ashx.) Accessed 20 November 2014.

College of Psychiatry of Ireland (2012). Submission to the Independent Monitoring Group on progress of A Vision for Change in 2011. (http://www.irishpsychiatry.ie/Libraries/External_Events_Documents/CPsychI_report_to_IM G_re_vision_in_2011_Final_3_February_2012_1.sflb.ashx). Accessed 20 November 2014.

Department of Education \& Skills (2015). Data on Individual Schools.

(https://www.education.ie/en/Publications/Statistics/Data-on-Individual-Schools/). Accessed 11 November 2015.

Department of Health \& Children (2006). A Vision for Change: Report of the Expert Group on Mental Health Policy. Government Publications Office: Dublin.

Flahavan C (2006). Detection, assessment and management of eating disorders; how involved are GPs? Irish Journal of Psychological Medicine 23, 96-99. 
Health Research Board (2014). HRB Statistics Series 25: Activities of Irish Psychiatric Units and Hospitals 2013. Dublin: Health Research Board.

(http://www.hrb.ie/uploads/tx_hrbpublications/HRB025_Statistics_Series_25_Activities_of_I rish_Psychiatric_Units_and_Hospitals_2013_online.pdf). Accessed_20 November 2014.

Health Service Executive (2011). National Mental Health Programme Plan.

(http://health.gov.ie/wp-

content/uploads/2014/04/National_Mental_Health_Programme_Plan_Nov2011_draft.pdf).

Accessed 4 June 2015.

McNicholas F, Dooley B, Keogh L, Lydon A, Lennon R, Ahern S, Coyle C, Whelan A, O'Donoghue L (2010). Eating problems in Irish children and adolescents - EPICA. Irish Journal of Psychological Medicine 27, 172-178.

McNicholas F, O'Connor C, O'Hara L, McNamara N (2015). Stigma and treatment of eating disorders in Ireland: Health professionals' knowledge and attitudes. Irish Journal of Psychological Medicine. Doi: http://dx.doi.org/10.1017/ipm.2015.24

O'Cathain A, Murphy E, Nicholl J (2007). Why, and how, mixed methods research is undertaken in health services research in England: A mixed methods study. BMC Health Services Research 7, 85.

Proctor EK, Landsverk J, Aarons G, Chambers D, Glisson C, Mittman B (2009). Implementation research in mental health services: An emerging science with conceptual, methodological, and training challenges. Administration and Policy in Mental Health 36, 2434.

Royal College of Psychiatrists (2012). Eating disorders in the UK: Service distribution, service development and training. Report from the Royal College of Psychiatrists Section of Eating Disorders. London: Royal College of Psychiatrists.

Strauss A, Corbin JM (1990). Basics of qualitative research: Grounded theory procedures and techniques. London: Sage. 